\title{
Fluctuations in Endogenous Kynurenic Acid Control Hippocampal Glutamate and Memory
}

\author{
Ana Pocivavsek', Hui-Qiu Wu', Michelle C Potter ${ }^{1,2}$, Greg I Elmer', Roberto Pellicciari ${ }^{3}$ and Robert Schwarcz ${ }^{*},{ }^{\prime}$ \\ 'Department of Psychiatry, Maryland Psychiatric Research Center, University of Maryland School of Medicine, Baltimore, MD, USA; \\ ${ }^{2}$ Department of Biological Sciences, University of Maryland Baltimore County, Baltimore, MD, USA; ${ }^{3}$ Dipartimento di Chimica e Tecnologia del \\ Farmaco, Universitá di Perugia, Perugia, Italy
}

\begin{abstract}
Kynurenic acid (KYNA), an astrocyte-derived metabolite, antagonizes the $\alpha 7$ nicotinic acetylcholine receptor ( $\alpha 7 \mathrm{nAChR})$ and, possibly, the glycine co-agonist site of the NMDA receptor at endogenous brain concentrations. As both receptors are involved in cognitive processes, KYNA elevations may aggravate, whereas reductions may improve, cognitive functions. We tested this hypothesis in rats by examining the effects of acute up- or downregulation of endogenous KYNA on extracellular glutamate in the hippocampus and on performance in the Morris water maze (MWM). Applied directly by reverse dialysis, KYNA (30-300 nM) reduced, whereas the specific kynurenine aminotransferase-II inhibitor (S)-4-(ethylsulfonyl)benzoylalanine (ESBA; 0.3-3 mM) raised, extracellular glutamate levels in the hippocampus. Co-application of KYNA (100 nM) with ESBA (I mM) prevented the ESBA-induced glutamate increase. Comparable effects on hippocampal glutamate levels were seen after intra-cerebroventricular (i.c.v.) application of the KYNA precursor kynurenine $(\mid \mathrm{mM}, 10 \mu \mathrm{l})$ or ESBA $(10 \mathrm{mM}, 10 \mu \mathrm{l})$, respectively. In separate animals, i.c.v. treatment with kynurenine impaired, whereas i.c.v. ESBA improved, performance in the MWM. I.c.V. co-application of KYNA $(10 \mu \mathrm{M})$ eliminated the pro-cognitive effects of ESBA. Collectively, these studies show that KYNA serves as an endogenous modulator of extracellular glutamate in the hippocampus and regulates hippocampus-related cognitive function. Our results suggest that pharmacological interventions leading to acute reductions in hippocampal KYNA constitute an effective strategy for cognitive improvement. This approach might be especially useful in the treatment of cognitive deficits in neurological and psychiatric diseases that are associated with increased brain KYNA levels.

Neuropsychopharmacology (20I I) 36, 2357-2367; doi: I0.1038/npp.20 I I. I27; published online 27 July 20 I I
\end{abstract}

Keywords: Alzheimer's disease; astrocytes; cognition; kynurenine; schizophrenia; water maze

\section{INTRODUCTION}

Kynurenic acid (KYNA), a metabolite of the kynurenine pathway of tryptophan degradation, is an endogenous antagonist of the $\alpha 7$ nicotinic acetylcholine receptor $(\alpha 7 \mathrm{nAChR})$ and the $N$-methyl-D-aspartate (NMDA) receptor (NMDAR), two ionotropic receptors with wellestablished links to cognitive functions (Levin et al, 2006; Robbins and Murphy, 2006; Thomsen et al, 2010). In mammals, KYNA is formed enzymatically by irreversible transamination of its direct bioprecursor, L-kynurenine ('kynurenine'). This process can be catalyzed by several distinct kynurenine aminotransferases (KATs), of which KAT-II is functionally best characterized in the brain (Guidetti et al, 2007a; Han et al, 2010). Thus, KAT-II is preferentially localized in astrocytes (Guidetti et al, $2007 \mathrm{~b}$ ), which readily release newly synthesized KYNA

*Correspondence: Dr R Schwarcz, Department of Psychiatry, Maryland Psychiatric Research Center, University of Maryland School of Medicine, PO Box 21247, Baltimore, MD 21228, USA, Tel: + I 410 402 7635, Fax: + I 4107472434 ,

E-mail: rschwarc@mprc.umaryland.edu

Received 24 February 2011; revised 6 June 2011; accepted I4 June 2011 into the extracellular milieu for possible inhibition of $\alpha 7 \mathrm{nAChRs}$ and NMDARs (Schwarcz and Pellicciari, 2002).

Relatively modest elevations of extracellular KYNA, caused by direct application of kynurenine or KYNA itself, or by systemic kynurenine-3-monooxygenase (KMO) inhibition, have remarkable biological consequences. Microdialysis studies in the striatum and the prefrontal cortex of awake rats showed that exogenous application of KYNA in the mid-nanomolar range, that is, 2-5 times endogenous levels, reduces the extracellular concentrations of dopamine and glutamate (Amori et al, 2009; Carpenedo et al, 2001; Moroni et al, 2005; Rassoulpour et al, 2005; Wu et al, 2010). Moreover, in the prefrontal cortex, nanomolar KYNA significantly decreases the amphetamine-induced stimulation of acetylcholine release (Zmarowski et al, 2009). Interestingly, pharmacological inhibition of KYNA synthesis with KAT-II inhibitors (Amori et al, 2009; Wu et al, 2010; Zmarowski et al, 2009) or genetic KAT-II elimination (Wu et al, 2007) has the opposite effects, that is, decreased KYNA production raises extracellular dopamine, glutamate, and acetylcholine levels. These results suggest that endogenous KYNA serves as a functionally significant neuromodulator in the mammalian brain. 
KYNA fluctuations may be especially consequential in the human brain, where KYNA levels are an order of magnitude higher than in rodents (Moroni et al, 1988; Turski et al, 1988). Moreover, KYNA levels are significantly elevated in the brain and the cerebrospinal fluid of individuals with schizophrenia, and in the brain of Alzheimer's disease patients, raising the possibility that enhanced inhibition of $\alpha 7 n A C h R s$ and NMDARs by KYNA has a causative role in the defining cognitive deficits seen in these diseases (Baran et al, 1999; Erhardt et al, 2001; Schwarcz et al, 2001). Indeed, acute systemic administration of kynurenine or peripheral KMO inhibition not only leads to increases in brain KYNA (Carpenedo et al, 1994; Russi et al, 1992; Swartz et al, 1990) but also causes distinct cognitive impairments in experimental animals (Chess and Bucci, 2006; Chess et al, 2007; 2009; Erhardt et al, 2004; Shepard et al, 2003). By contrast, we showed recently that chronic reduction of brain KYNA in KAT-II-knockout mice improves cognitive performance (Potter et al, 2010). Notably, the latter study also included microdialysis in the hippocampus, a brain area critically implicated in several important aspects of cognitive processing and abundantly endowed with both $\alpha 7 n A C h R s$ and NMDARs (Fabian-Fine et al, 2001; Monaghan et al, 1989; Morris, 2006). Our data demonstrated that the reduction in basal KYNA levels in mutant mice was accompanied by elevated extracellular glutamate levels. This increase in glutamate might be causally related to the cognitive improvements seen in the KYNA-deficient animals (Robbins and Murphy, 2006).

The possible pro-cognitive consequences of an acute reduction in hippocampal KYNA formation have not been examined so far. In addition, no data are currently available on the effects of raised KYNA levels on an animal's performance in the Morris water maze (MWM), a classic spatial learning and memory task involving the hippocampus and associated brain regions (Morris, 1984; Jo et al, 2007). The present study was designed to fill this void using experimental rats. In separate animals, we re-assessed the effects of KYNA on extracellular glutamate levels in the hippocampus (Moroni et al, 2005).

\section{MATERIALS AND METHODS}

\section{Animals}

Adult, male Sprague-Dawley rats (300-400 g) were used in all experiments. The animals were housed in a temperaturecontrolled facility at the Maryland Psychiatric Research Center. They were kept on a 12/12-h light-dark cycle and had free access to food and water. The facility was fully accredited by the American Association for the Accreditation of Laboratory Animal Care, and the experimental protocols followed the 'Principles of Laboratory Animal Care' (NIH publication no. 86-23, 1996).

\section{Chemicals}

KYNA and glutamate were purchased from Sigma (St Louis, MO, USA). L-Kynurenine sulfate ('kynurenine'; purity: 99.4\%) was obtained from Sai Adventium (Hyderabad, India). (S)-4-(ethylsulfonyl)benzoylalanine (ESBA) was synthesized as described by Pellicciari et al (2008). Other chemicals were obtained from a variety of suppliers and were of the highest commercially available purity. For intracerebral injections, all test compounds were dissolved in sterile, phosphate-buffered saline (PBS).

\section{Microdialysis}

Rats were anesthetized with chloral hydrate $(360 \mathrm{mg} / \mathrm{kg}$, i.p.) and mounted in a David Kopf stereotaxic frame. A guide cannula (outer diameter: $0.65 \mathrm{~mm}$ ) was positioned over the dorsal hippocampus (AP: $-3.4 \mathrm{~mm}$ from bregma; L: $2.3 \mathrm{~mm}$; $\mathrm{V}: 1.5$ below dura) and secured to the skull with anchor screws and acrylic dental cement. On the next day, a microdialysis probe (CMA/10, membrane length: $2 \mathrm{~mm}$; Carnegie Medicin, Stockholm, Sweden) was inserted through the guide, protruding vertically through the hippocampus. The probe inlet was connected to a microperfusion pump (CMA/100; Carnegie Medicin) set to a speed of $1 \mu \mathrm{l} / \mathrm{min}$, and the freely moving animals were perfused with Ringer solution $(\mathrm{pH}$ 6.7) containing $144 \mathrm{mM} \mathrm{NaCl}, 4.8 \mathrm{mM} \mathrm{KCl}, 1.2 \mathrm{mM} \mathrm{MgSO}_{4}$, and $1.7 \mathrm{mM} \mathrm{CaCl}_{2}$. Thirty-minute fractions were collected for the duration of the experiment. The first sample was discarded and baseline values were collected during the next $2 \mathrm{~h}$ (four fractions), prior to the experimental intervention. Test compounds, dissolved in Ringer solution, were administered by reverse dialysis directly into the hippocampus.

Microdialysis data were not corrected for recovery from the dialysis probe.

\section{I.c.v. Infusion}

In preparation of an intra-cerebroventricular (i.c.v.) infusion, rats were anesthetized as above, placed in a stereotaxic apparatus, and implanted with a 22-gauge guide cannula that extended into the lateral ventricle (coordinates: AP: $+0.9 \mathrm{~mm}$ from bregma; L: $1.4 \mathrm{~mm}$; V: $2.1 \mathrm{~mm}$ below dura). On the day of the experiment, test compounds or vehicle (PBS) were infused (10 $\mu \mathrm{l}$ in $5 \mathrm{~min})$ through a 28 -gauge injection needle that was inserted through the guide and extended $1.0 \mathrm{~mm}$ beyond the tip of the guide. Infusion was controlled by a CMA/100 microinfusion pump.

Where indicated, microdialysis was performed in the hippocampus contralateral to the i.c.v. infusion.

\section{KYNA and Glutamate Determination}

The content of KYNA and glutamate in microdialysate was determined according to established high-performance liquid chromatography (HPLC) procedures. Briefly, KYNA was measured following isocratic elution with a mobile phase containing $200 \mathrm{mM}$ zinc acetate and $5 \%$ acetonitrile ( $\mathrm{pH}$ 6.2), and detected fluorimetrically (excitation wavelength: $344 \mathrm{~nm}$; emission wavelength: 398) (Shibata, 1988). Glutamate (o-phthalaldehyde/2-mercaptoethanol derivatization; excitation wavelength: $390 \mathrm{~nm}$; emission wavelength: $460 \mathrm{~nm})$ was determined fluorimetrically after gradient elution (Quarta et al, 2004).

\section{Behavioral Testing}

Animals were cannulated for i.c.v. infusion and were then given at least 5 days to recover from surgery. On the day of 
the experiment, vehicle (PBS) or test compounds were administered i.c.v. $90 \mathrm{~min}$ prior to behavioral testing.

The MWM was used to study spatial navigation and reference memory (Morris, 1984). The maze pool was $180 \mathrm{~cm}$ in diameter, filled with $20-22^{\circ} \mathrm{C}$ water made opaque with non-toxic white tempura paint, and surrounded by distinct extra-maze spatial cues. To habituate, each rat was allowed to navigate the pool for $120 \mathrm{~s}$. On the first day of training, a hidden platform $(10 \mathrm{~cm}$ in diameter, approximately $2 \mathrm{~cm}$ below water level) was placed in the $\mathrm{NW}$ quadrant and remained there across training trials (4 each day) and days (4 consecutive days). Each day, each rat was tested in four trials that were initiated at the NW, NE, SW, and SE quadrants, respectively. Start location was run in a counterbalanced order across trials and animals. Each animal was given up to $120 \mathrm{~s}$ to locate the hidden platform. Upon finding the platform, the animal was required to stay on it for $15 \mathrm{~s}$. If an animal did not find the platform, it was gently guided to the platform location and made to stay on the platform for $15 \mathrm{~s}$. The inter-trial interval was $120 \mathrm{~s}$. On the fifth day, the hidden platform was removed. The animal was then given $120 \mathrm{~s}$ to navigate the pool, allowing us to test retention and spatial navigation strategy ('probe trial'). Immediately following the probe trial, each animal was tested again, with the platform made visible by a red flag and raised slightly above the water level ('visible trial'). This trial was used to monitor visual acuity and general sensorimotor capabilities. Behavior during all trials was recorded by using a Noldus EthoVision tracking system (Leesburg, VA, USA).

At the end of behavioral testing, $10 \mu \mathrm{l}$ of a $1 \%$ cresyl violet solution were infused i.c.v. The animals were then killed and their brain was sectioned on a cryostat to verify the presence of dye in the ventricle. Only animals with proper placement of the guide cannula were included in the data analysis.

\section{Statistical Analysis}

Two-group comparisons were made using an unpaired Student's $t$-test. Three or more groups were compared by one-way ANOVA with appropriate post-hoc analysis. A two-way, repeated-measures (RM) ANOVA with appropriate post-hoc analysis (indicated in section Results or in the figure legends) was used to determine how a response was influenced by two factors. Statistical significance was defined as $P<0.05$.

\section{RESULTS}

\section{Nanomolar KYNA Reduces Extracellular Glutamate Levels in the Hippocampus}

In the first set of experiments, we tested whether KYNA, applied by reverse dialysis, would impact extracellular glutamate levels within the hippocampus. The basal level of extracellular glutamate, collated from all 46 rats undergoing hippocampal microdialysis in the course of the study, was $2.2 \pm 0.1 \mu \mathrm{M}$. Local perfusion of $100 \mathrm{nM}$ and $300 \mathrm{nM}$ KYNA caused dose-dependent reductions in glutamate, resulting in a nadir of $64 \%$ and $58 \%$, respectively, of baseline levels after $2 \mathrm{~h}(P<0.05 ; n=5$ per group; two-way ANOVA with
Bonferroni's post-hoc analysis). Glutamate levels returned to basal values following the removal of KYNA from the perfusion solution. Perfusion with a lower concentration of KYNA $(30 \mathrm{nM})$ failed to reduce extracellular glutamate levels (Figure 1).

\section{Reduction of KYNA Synthesis Enhances Extracellular Glutamate Levels in the Hippocampus}

We next examined the effects of decreased KYNA formation on extracellular glutamate levels in the hippocampus. To this end, the specific KAT-II inhibitor ESBA was applied by reverse dialysis for $2 \mathrm{~h}$ and the concentrations of KYNA and glutamate were determined in the microdialysate. The basal level of extracellular KYNA, collated from all 21 rats undergoing hippocampal microdialysis for the measurement of KYNA in the course of the study, was $2.5 \pm 0.2 \mathrm{nM}$. ESBA dose-dependently reduced extracellular KYNA and increased extracellular glutamate levels (Figure 2a and b; $n=4$ per group; two-way ANOVA with Bonferroni's posthoc analysis). At the highest concentration used ( $3 \mathrm{mM})$, ESBA application caused a maximal reduction to $69 \%$ of baseline KYNA levels and a maximal increase to $333 \%$ of baseline glutamate values after $2 \mathrm{~h}(P<0.05$ each). Both KYNA and glutamate levels gradually returned to baseline values after ESBA was removed from the perfusion solution.

In separate rats $(n=5)$, ESBA $(1 \mathrm{mM})$ was co-applied with $100 \mathrm{nM}$ KYNA under otherwise identical experimental conditions. In these animals, extracellular glutamate levels did not differ to an important degree from baseline levels throughout the $2-h$ perfusion period $(P>0.05$; Figure $2 c)$. Immediately following the infusion of ESBA + KYNA (ie, at the 2.5 -h time point), glutamate levels reached a maximum of $114 \%$ of baseline values, but this elevation was not statistically different from baseline $(P=0.41$; Student's $t$-test). Moreover, the magnitude of this change was much less than the increase in glutamate levels seen with ESBA alone (cf. Figure 2b). In other words, the ESBA-induced

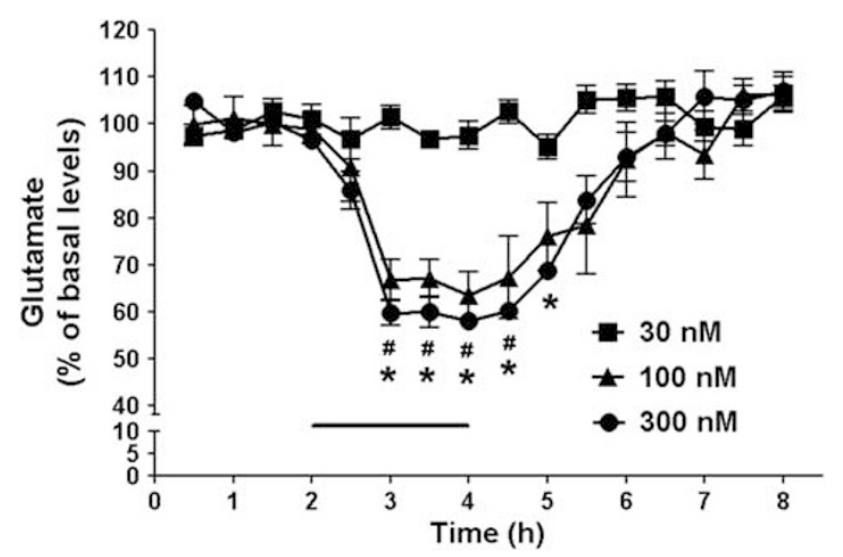

Figure I Dose-dependent effect of KYNA on extracellular levels of glutamate in the hippocampus. After $2 \mathrm{~h}$ of baseline collection, KYNA was applied intra-hippocampally for $2 \mathrm{~h}$ by reverse dialysis (bar). Data are expressed as a percentage of baseline (see Results for absolute value) and are the mean \pm SEM ( $n=5$ per group). ${ }^{\#}$ and ${ }^{*} P<0.05$ vs baseline for 100 and $300 \mathrm{nM} \mathrm{KYNA}$, respectively (two-way ANOVA with Bonferroni's posthoc test). 


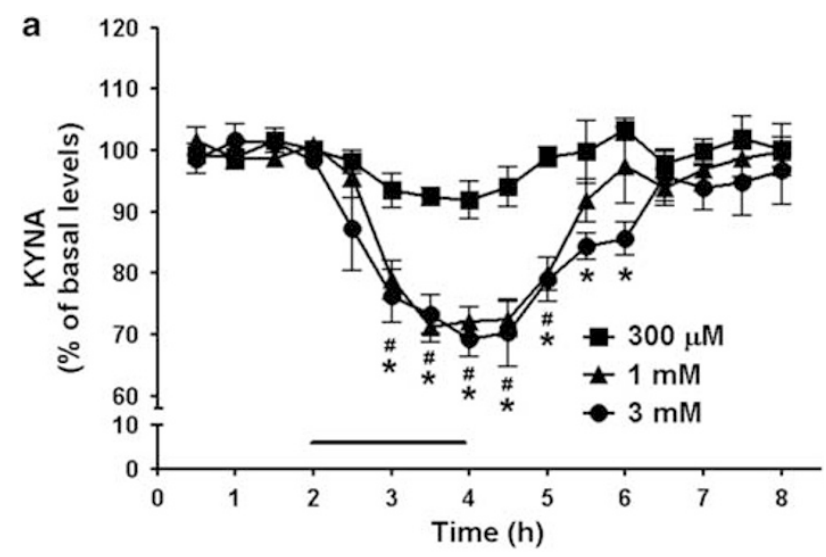

increased concentration of glutamate measured in the microdialysate.
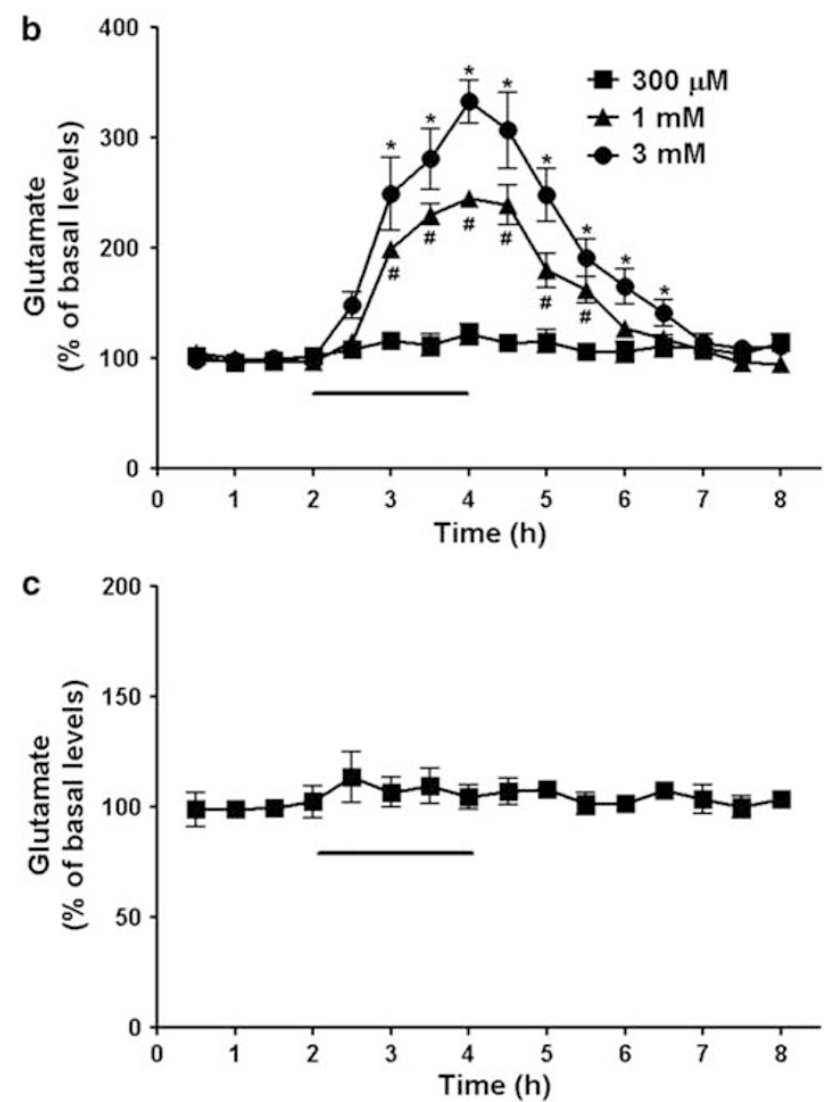

\section{I.c.v. Infusion of Kynurenine or ESBA: Neurochemical Effects in the Contralateral Hippocampus}

Fluctuations in extracellular glutamate levels in the hippocampus may affect cognitive processes (Richter-Levin et al, 1995). In preparation of behavioral studies (see below), we therefore tested the neurochemical sequelae of acute increases or reductions in endogenous KYNA levels by infusing the bioprecursor kynurenine and ESBA, respectively, i.c.v. This route of administration was chosen to allow bilateral distribution of the test compounds and minimize tissue damage. Following the establishment of optimal treatment regimens (data not shown), solutions of kynurenine $(1 \mathrm{mM})$ or ESBA $(10 \mathrm{mM})$ were infused unilaterally $(10 \mu \mathrm{l}$ over $5 \mathrm{~min})$, and microdialysis was performed concomitantly in the contralateral hippocampus to determine extracellular levels of both KYNA and glutamate.

Infusion of kynurenine caused a $346 \%$ increase in extracellular KYNA and a concomitant reduction in extracellular glutamate (nadir: $72 \%$ of baseline values) $(P<0.05$ each; $n=4$; two-way ANOVA with Bonferroni's post-hoc analysis). These effects were transient, and both KYNA and glutamate levels returned to basal levels by $4 \mathrm{~h}$ (Figure 3a). Conversely, extracellular KYNA levels were reduced by a unilateral infusion of ESBA (nadir: $73 \%$ of baseline values), whereas glutamate levels simultaneously increased, reaching a maximum of $177 \%$ of baseline values $(P<0.05$ each; $n=5$; two-way ANOVA with Bonferroni's post-hoc analysis). These changes, too, were transient (Figure 3b).

We next investigated whether the increase in extracellular glutamate caused by an i.c.v. infusion of ESBA could be attenuated by normalizing KYNA levels. To this end, KYNA $(10 \mu \mathrm{M})$ was co-applied with ESBA $(10 \mathrm{mM})$ ('ESBA + KYNA'). Infusion of ESBA + KYNA did not affect extracellular glutamate levels in the contralateral hippocampus (Figure 3c), indicating that the increase in extracellular glutamate caused by ESBA alone had been secondary to a reduction in KYNA levels.

Figure 2 Dose-dependent effects of ESBA on extracellular KYNA and glutamate levels in the hippocampus. After $2 \mathrm{~h}$ of baseline collection, ESBA was applied intra-hippocampally for $2 \mathrm{~h}$ by reverse dialysis (bar). KYNA (a) and glutamate (b) were measured in the same dialysate. Data are expressed as a percentage of baseline values and are the mean \pm SEM ( $n=4$ per group). " and $* P<0.05$ vs baseline for 1 and $3 \mathrm{mM} \mathrm{ESBA}$, respectively (two-way ANOVA with Bonferroni's post-hoc test). (c) After $2 \mathrm{~h}$ of baseline collection, ESBA (I mM) and KYNA (I00 nM) were co-infused into the hippocampus for $2 \mathrm{~h}$ (bar). Data are expressed as a percentage of baseline values and are the mean \pm SEM $(n=5)$. Perfusion with ESBA + KYNA did not affect extracellular glutamate levels $(P>0.05$; two-way ANOVA with Bonferroni's post-hoc test). Absolute baseline values of KYNA and glutamate are given under Results.

increase in extracellular glutamate could be neutralized by re-introducing a low concentration of KYNA into the extracellular milieu. These data corroborated causality between the reduction in KYNA neosynthesis and the

\section{Acute Elevation of KYNA Impairs Performance in the MWM}

Next, we tested the effect of an acute KYNA elevation in the MWM. The data are shown as the average of the four trials (different start locations) for all animals (Figure 4a). Compared with vehicle-treated controls, kynurenine treatment produced an overall significant effect on escape latency across days $\left(\mathrm{F}_{1,22}=6.25, n=12\right.$ per group; $P<0.05$; two-way RM ANOVA). On days 2,3 , and 4 of training, kynurenine-treated animals significantly differed from the controls with respect to escape latency $(P<0.05$; post-hoc Student's $t$-test; Figure $4 \mathrm{a})$. The two treatment groups did not differ significantly in escape latency on day 1 , and no significant differences were seen among individual trials on day 1 (Supplementary Figure 1), suggesting that kynurenine treatment produced a learning deficit across days that was not driven solely by performance on the first day of testing. 

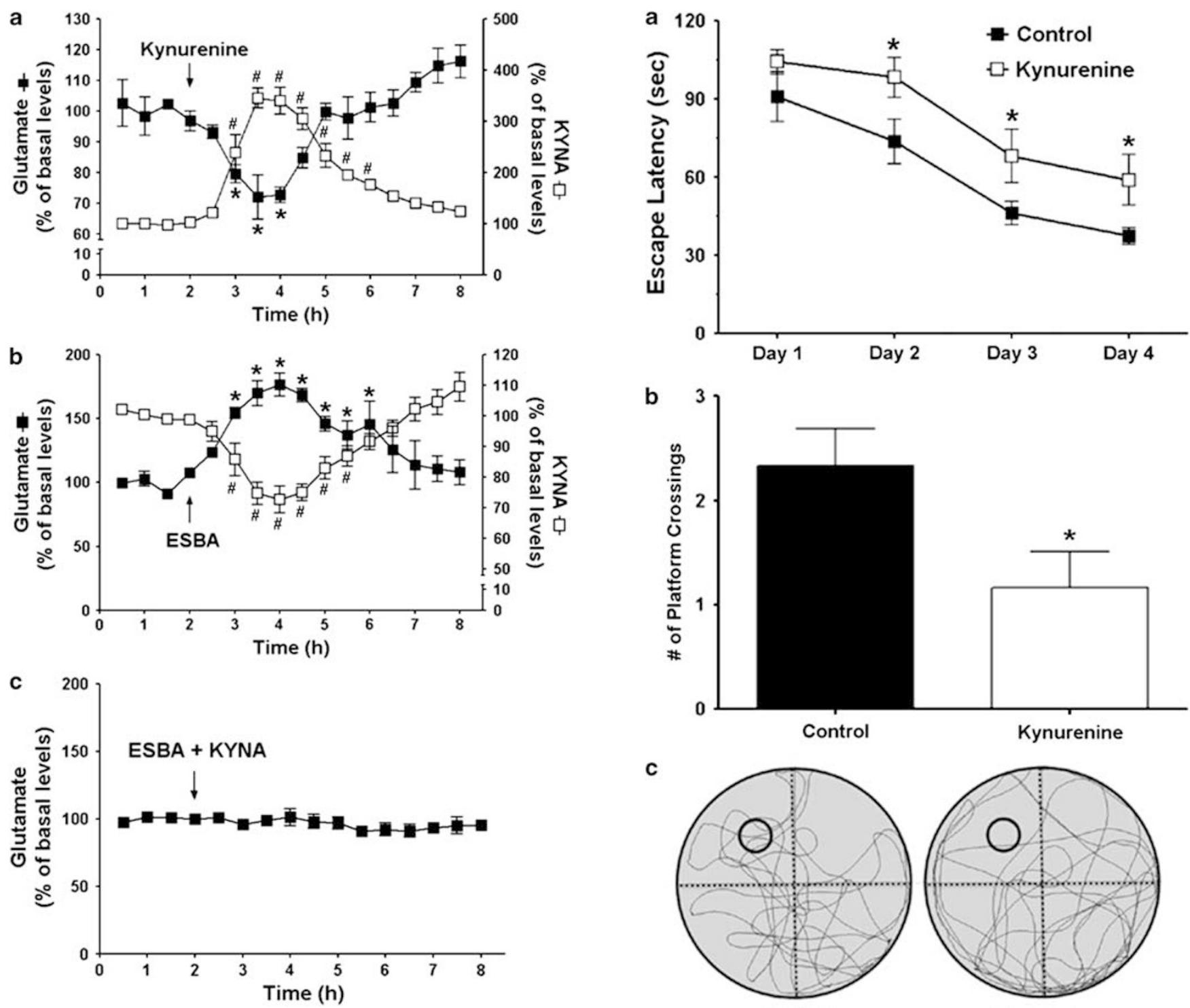

c

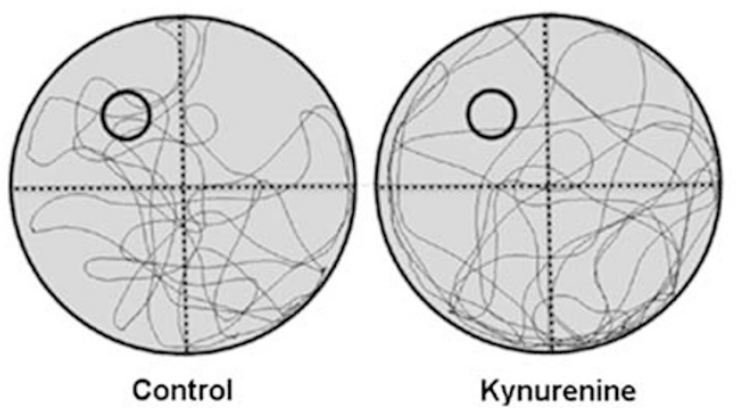

Figure 3 Acute, unilateral i.c.v. infusion of kynurenine or ESBA affects neurochemistry in the contralateral hippocampus. After $2 \mathrm{~h}$ of baseline collection, test compounds were applied i.c.v. (a) kynurenine (I mM); (b) ESBA $(10 \mathrm{mM}) ;(\mathrm{c}) \operatorname{ESBA}(10 \mathrm{mM})+\mathrm{KYNA}(10 \mu \mathrm{M}))$ (arrows) and the microdialysate was collected from the contralateral hippocampus. KYNA and glutamate were measured in the same dialysate where applicable. Data are expressed as a percentage of baseline (see Results for absolute values) and are the mean $\pm \operatorname{SEM}(n=4$ for kynurenine; $n=5$ for ESBA; $n=5$ for ESBA + KYNA). ${ }^{\#}$ and $* P<0.05$ vs the respective baseline (two-way ANOVA with Bonferroni's post-hoc test).

Retention of the newly learned task and spatial navigation strategy was assessed in one single probe trial $24 \mathrm{~h}$ after the last training session. Animals treated with kynurenine crossed the area formerly occupied by the platform significantly less frequently than vehicle-treated animals (control: $2.3 \pm 0.4$, kynurenine: $1.2 \pm 0.3 ; P<0.05$; Student's $t$-test) (Figure $4 \mathrm{~b}$ ). Representative images of the swim paths of the controls and the kynurenine-treated animals during the probe trial are shown in Figure 4c. During the 120-s swim period in the probe trial, the kynurenine-treated animals also spent significantly less time in the target NW quadrant than the vehicle-treated controls (Supple-

mentary Figure $2 \mathrm{~A}$ ). The probe trial was further analyzed in 30-s epochs. This analysis revealed that kynureninetreated animals, compared with the vehicle-treated controls, spent significantly less time in close proximity to the former platform location during the first $30 \mathrm{~s}$ (Supplementary Figure 2B). However, there were no significant differences between the two groups in subsequent 30-s epochs, suggesting that animals searched all quadrants unsuccessfully after not finding the platform within the first $30 \mathrm{~s}$. Swim speed (control: $28.3 \pm 1.6 \mathrm{~cm} / \mathrm{s}$, kynurenine: $28.9 \pm 2.2 \mathrm{~cm} / \mathrm{s}$ ) did not differ significantly between the two groups. Furthermore, the escape latencies of the 
vehicle- and the kynurenine-treated animals did not differ on the visible trial, which was conducted immediately after the probe trial (control: $17.6 \pm 4.0 \mathrm{~s}$, kynurenine: $14.7 \pm 3.0 \mathrm{~s}$ ), suggesting that kynurenine treatment did not cause gross visual deficits. Taken together, our data showed that elevating endogenous KYNA levels in the hippocampus impaired performance in a hippocampus-mediated learning and memory task.

\section{Acute Reduction of KYNA Formation Improves Performance in the MWM}

In separate animals, we tested whether inhibition of KYNA synthesis affects performance in the MWM. Compared with rats receiving vehicle, ESBA treatment produced an overall significant effect on escape latency across days $\left(\mathrm{F}_{1,22}=5.64\right.$; $n=12$ per group; $P<0.05$; two-way RM ANOVA). Post-hoc analysis revealed a significant reduction in escape latency on days 1 and 2 in animals treated with ESBA $(P<0.05$; Student's $t$-test) (Figure 5a). The difference on the first day of acquisition training was caused by the fact that the ESBAtreated animals improved across individual trials on day 1 (Supplementary Figure 3). However, the two treatment groups did not differ significantly in the escape latency of the first trial (Supplementary Figure 3), demonstrating that both groups began acquisition training with similar performance.

To assess retention and navigation strategy after 4 days of MWM training, a single probe trial was performed $24 \mathrm{~h}$ after the last training session. Animals treated with ESBA crossed the former platform location significantly more often than the vehicle-treated animals (control: $2.0 \pm 0.3$, ESBA: $3.6 \pm 0.6$; Student's $t$-test; $P<0.05$ ) (Figure $5 \mathrm{~b}$ ). Representative images of the swim paths of the vehicle- and the ESBAtreated animals during the probe trial are depicted in Figure 5c. During the probe trial, the ESBA-treated rats also spent significantly more time in the target NW quadrant than the vehicle-treated animals (Supplementary Figure $4 \mathrm{~A})$. Further analysis revealed that ESBA-treated animals, compared with vehicle-treated animals, spent significantly more time in close proximity to the former platform location during the first 30-s epoch (Supplementary Figure 4B). However, there were no significant differences between the two groups in subsequent 30 -s epochs, suggesting that animals searched all quadrants unsuccessfully after not finding the platform within the first $30 \mathrm{~s}$. Furthermore, swim speed (control: $28.5 \pm 1.1 \mathrm{~cm} / \mathrm{s} v s$ ESBA $26.2 \pm 1.3 \mathrm{~cm} / \mathrm{s}$ ) did not differ significantly between the treatment groups. Moreover, the escape latencies of the controls and the animals treated with the KAT-II inhibitor did not differ on the visible trial immediately after the probe trial (control: $12.9 \pm 2.3 \mathrm{~s}$, ESBA: $14.7 \pm 2.4 \mathrm{~s}$ ), indicating that ESBA did not cause any gross visual deficits. Thus, specific inhibition of KYNA synthesis in the brain acutely induced pro-cognitive effects in the rodents tested in the MWM.

\section{Co-application of KYNA Neutralizes the Pro-cognitive Effects of KAT-II Inhibition}

To test whether normalization of KYNA levels prevents the pro-cognitive effects of ESBA, ESBA $(10 \mathrm{mM})$ and KYNA $(10 \mu \mathrm{M})$ were jointly administered i.c.v. and performance in


Figure 5 Acute i.c.v. infusion of ESBA improves spatial memory. (a) Vehicle (control) or ESBA $(10 \mathrm{mM})$ were infused i.c.v. $90 \mathrm{~min}$ prior to MWM testing each day. See text for experimental details. The data are the mean \pm SEM ( $n=12$ per group). $* P<0.05$ vs control (post-hoc Student's t-test). (b) Number of platform crossings during the probe trial (video tracking analysis). The data are the mean \pm SEM ( $n=12$ per group). * $P<0.05$ vs control (Student's t-test). (c) Representative swim path traces for the probe trial. The former platform location is indicated by small black circles.

the MWM was compared with animals receiving i.c.v. ESBA only ( $n=12$ for ESBA alone and $n=11$ for ESBA + KYNA). A new group of vehicle-treated animals was also tested in these experiments to serve as an internal control $(n=12)$. A two-way RM ANOVA revealed a significant main effect of treatment (control, ESBA, ESBA + KYNA) $\left(\mathrm{F}_{2,32}=4.74\right)$. Pairwise comparison confirmed our previous finding that ESBA significantly improved performance in the MWM $\left(\mathrm{F}_{1,22}=4.99 ; P<0.05\right.$; two-way RM ANOVA $)$. Co-application of ESBA and KYNA produced an overall significant 

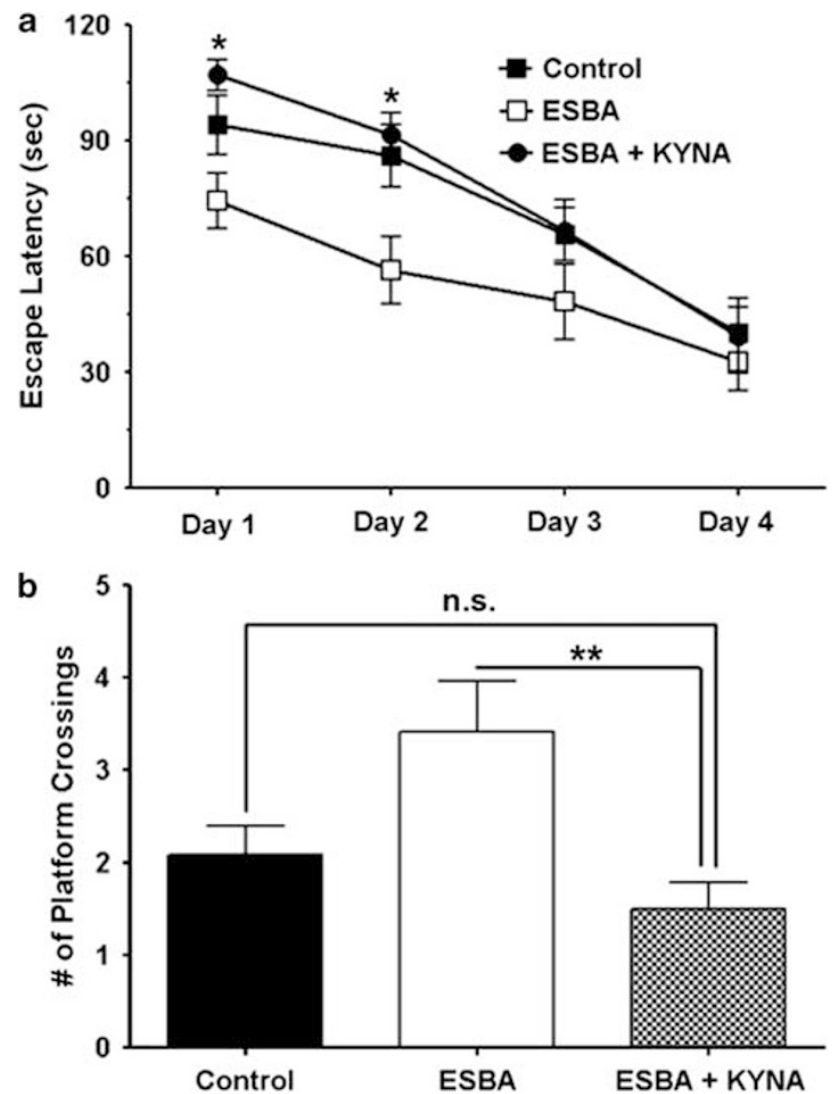

C

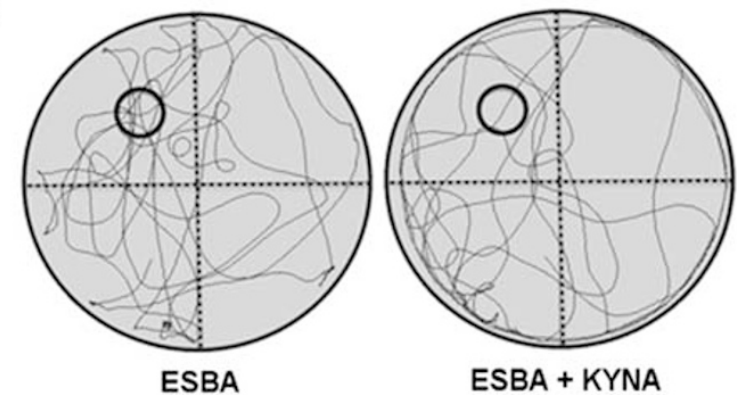

Figure 6 Acute i.c.V. co-infusion of KYNA prevents the ESBA-induced cognitive enhancement. (a) Vehicle (control), ESBA (I0 mM), or ESBA $(10 \mathrm{mM})+\mathrm{KYNA}(10 \mu \mathrm{M})$ was infused i.c.v. 90 min prior to MWM testing each day. See text for experimental details. The data are the mean \pm SEM ( $n=12$ for control; $n=12$ for ESBA; $n=11$ for ESBA + KYNA). *P $<0.05$ (ESBA + KYNA vs ESBA alone; post-hoc Student's t-test). (b) Number of platform crossings during the probe trial (video tracking analysis). The data are the mean \pm SEM ( $n=12$ for control; $n=12$ for ESBA; $n=11$ for ESBA $+K Y N A) .{ }^{*} * P<0.0$ I, n.s.: not significant $(P>0.05)$ (Student's $t$-test). (c) Representative swim path traces for the probe trial. The former platform location is indicated by small black circles.

effect on escape latency across days when compared with ESBA treatment alone $\left(\mathrm{F}_{1,21}=7.60 ; P<0.05\right.$; two-way $\mathrm{RM}$ ANOVA). Post-hoc analysis revealed a significant increase in escape latency on days 1 and 2 in animals treated with ESBA + KYNA as compared with animals treated with ESBA alone $(P<0.05$; Student's $t$-test) (Figure 6a). The animals treated with ESBA + KYNA did not, however, differ significantly with respect to escape latency across days, when compared with the vehicle-treated animals $\left(\mathrm{F}_{1,21}=0.42 ; P>0.05\right)$. The significant difference in escape latency on the first day of acquisition training was related to the fact that the ESBAtreated animals improved across individual trials on day 1 (cf. also Supplementary Figure 3), whereas animals treated with ESBA + KYNA did not improve (Supplementary Figure 5). Notably, the three treatment groups did not differ significantly in escape latency in the first trial, that is, all treatment groups began acquisition training with similar performance (Supplementary Figure 5). Taken together, unlike animals treated with ESBA alone, rats treated with ESBA + KYNA did not show improved performance in the MWM. Thus, the pro-cognitive effect of ESBA could be traced to the compound's ability to reduce endogenous KYNA production.

Retention of the newly formed memory and spatial navigation strategy were assessed in a probe trial $24 \mathrm{~h}$ after the last training session. Animals treated with ESBA+ KYNA crossed the location formerly occupied by the platform significantly less often than animals receiving ESBA alone (ESBA: $3.4 \pm 0.5$, ESBA + KYNA: $1.5 \pm 0.3 ; P<0.01$; Student's $t$-test) (Figure $6 \mathrm{~b}$ ). With respect to platform crossings, the KYNA + ESBA-treated animals did not differ significantly from the vehicle-treated animals (control: $2.1 \pm 0.3$ ). Representative images of the swim paths of rats receiving ESBA + KYNA or ESBA during the probe trial are shown in Figure $6 c$. These images indicate that animals treated with ESBA+KYNA spent less time in the NW quadrant and around the platform location than animals treated with ESBA alone. On average, ESBA + KYNA-treated rats (similar to vehicle-treated animals) also spent less time in the target NW quadrant than animals treated with ESBA alone (Supplementary Figure 6A). Further analysis revealed that animals treated with ESBA + KYNA spent significantly less time in close proximity to the former platform location during the first 30 -s epoch of the probe trial when compared with ESBA-treated rats (Supplementary Figure 6B). However, there were no significant differences between the three groups in subsequent 30-s epochs, suggesting that animals searched all quadrants unsuccessfully after not finding the platform within the first $30 \mathrm{~s}$. Swim speed (ESBA: $29.6 \pm 1.6 \mathrm{~cm} / \mathrm{s}$, ESBA + KYNA: $30.9 \pm 0.9 \mathrm{~cm} / \mathrm{s}$, control: $29.1 \pm 0.8 \mathrm{~cm} / \mathrm{s}$ ) did not differ significantly between the three groups. Finally, the escape latencies of animals in the three groups did not differ on the visible platform trial (ESBA: $15.2 \pm 3.1 \mathrm{~s}$, ESBA + KYNA: $16.0 \pm 2.6 \mathrm{~s}$, control: $13.3 \pm 2.5 \mathrm{~s}$ ), indicating that drug treatment did not cause gross visual deficits. Thus, normalization of KYNA levels returned performance in the MWM to that of vehicle-treated control animals.

\section{DISCUSSION}

We showed here that acute fluctuations in brain KYNA bi-directionally control extracellular glutamate levels in the rat hippocampus. We also established, in separate animals, that elevation in cerebral KYNA levels aggravates, whereas inhibition of KYNA formation improves, performance in the MWM, a well-established assay for spatial learning and memory. Using both chemical and behavioral endpoints, we then demonstrated that neither the rise in glutamate nor the cognitive improvement seen after the administration of the KYNA synthesis inhibitor ESBA were observed when the 
compound's effect was neutralized by the addition of exogenous KYNA. These results, taken together, demonstrate that up- and downregulation of endogenous KYNA in the brain acutely modulate hippocampal chemistry and function in vivo. Our findings confirm and extend reports of similar glutamate changes following KYNA manipulations in other forebrain regions (Carpenedo et al, 2001; Konradsson-Geuken et al, 2009; Wu et al, 2010), and are also in line with experiments showing cognitive impairments following systemic administration of kynurenine, the immediate bioprecursor of KYNA (Chess and Bucci, 2006; Chess et al, 2007; 2009; Erhardt et al, 2004; Shepard et al, 2003). Most importantly, the present data provide the first demonstration that the cognitive improvement seen in animals with genetic, that is, chronic, elimination of KAT-II (Potter et al, 2010) can be duplicated by acute, specific KAT-II inhibition.

Originally introduced as a nonspecific, 'broad-spectrum' antagonist of ionotropic excitatory amino-acid receptors at high micromolar concentrations (Perkins and Stone, 1982), KYNA is now known to specifically target the glycine coagonist site of the NMDAR (Kessler et al, 1989) and the $\alpha 7 \mathrm{nAChR}$ (Hilmas et al, 2001) at concentrations that are much closer to ambient brain levels. Although endogenous KYNA can also activate the G-protein-coupled receptor GPR35 (Cosi et al, 2010; Wang et al, 2006), inhibition of $\alpha 7$ nAChRs and, possibly, NMDARs appears to be the primary physiological function of extracellular KYNA in the mammalian brain (see below). As both $\alpha 7 n A C h R s$ and NMDARs are critically involved in many important physiological functions, including cognitive processes (Bannerman et al, 2006; Levin et al, 2006; Robbins and Murphy, 2006; Thomsen et al, 2010), and also have a role in the etiology of neurodegenerative and other catastrophic brain diseases (Kalia et al, 2008; Kantrowitz and Javitt, 2010; Martin and Freedman, 2007; Mudo et al, 2007), detection of KYNA in the mammalian brain (Moroni et al, 1988; Turski et al, 1988) immediately suggested an important role of the metabolite in both physiology and pathology (Pereira et al, 2002; Schwarcz et al, 1992). The idea was reinforced by the discovery of specific mechanisms controlling KYNA synthesis in the brain (Gramsbergen et al, 1997) and, in particular, by reports of abnormal KYNA levels in nervous tissue and body fluids in a host of neurological and psychiatric disorders (for review see Chen et al, 2009; Nemeth et al, 2005). Several of these diseases, including schizophrenia (Erhardt et al, 2001; Schwarcz et al, 2001), depression (O'Connor et al, 2009; Raison et al, 2010), and Alzheimer's disease (Baran et al, 1999), also show distinct hippocampal pathology, which is likely responsible for many of the defining cognitive deficits (Barnes et al, 2009; Scheff and Price, 2006; Tamminga et al, 2010). It remains to be seen, however, whether and how anomalies in KYNA metabolism or function might be causally involved in the pathological processes.

In vivo studies in experimental animals, as well as work using cells or tissue slices in vitro, revealed that the irreversible transamination of kynurenine to KYNA in the brain can be catalyzed by four KATs (Guidetti et al, 2007a; Han et al, 2010). Although several of these enzymes may participate in cerebral KYNA biosynthesis under physiological and pathological conditions, it appears that the pool of KYNA that can be most readily mobilized in the brain is largely provided by KAT-II (Amori et al, 2009). This enzyme is almost exclusively localized in astrocytes (Guidetti et al, 2007b; Guillemin et al, 2001), which rapidly liberate newly synthesized KYNA into the extracellular milieu (Curatolo et al, 1996; Guillemin et al, 2000). Although the precise mechanism controlling the release of KYNA has not been elucidated, this insight led to the development of specific KAT-II inhibitors, which are designed to target astrocytes and reduce extracellular KYNA concentrations. From the outset, these agents were not only viewed as tools to study the neurobiology of KYNA, but were also appreciated for their potential therapeutic applications (Schwarcz and Pellicciari, 2002; Varasi et al, 1996). ESBA, which was used in the present study, was introduced as the first specific KAT-II inhibitor in 2006 (Pellicciari et al, 2006).

The fact that intracerebral application of kynurenine and ESBA, respectively, up- and downregulated KYNA production in the hippocampus, is consistent with previous studies in other regions of the rat brain (Amori et al, 2009; Speciale et al, 1990; Zmarowski et al, 2009). Also in agreement with published studies (Konradsson-Geuken et al, 2009; Wu et al, 2010), we found that these manipulations have secondary effects on extracellular glutamate in the hippocampus. We did, however, fail to confirm the report that extracellular glutamate is not reduced following intra-hippocampal KYNA infusion (Moroni et al, 2005). This indicates that modulation by KYNA may constitute an additional general mechanism by which astrocytes influence glutamatergic neurotransmission in the brain (Hamilton and Attwell, 2010; Hertz and Zielke, 2004; Parpura and Zorec, 2010).

Both anatomical and pharmacological studies suggest that the bidirectional effects of KYNA on glutamate in the hippocampus involve $\alpha 7 \mathrm{nAChRs}$ as a primary target. Thus, the extracellular levels of KYNA attained in the present study were in the low-to-mid-nanomolar range, sufficient to inhibit $\alpha 7 \mathrm{nAChRs}$ (Hilmas et al, 2001) but less likely to reach the concentrations required to inhibit NMDARs directly (Kessler et al, 1989; Parsons et al, 1997). In the hippocampus as elsewhere (Albuquerque et al, 2009), $\alpha 7 n A C h R s$ are prominently associated with nerve terminals, where they can be targeted to modulate glutamate release (Fabian-Fine et al, 2001; Gray et al, 1996; Lagostena et al, 2008). $\alpha 7 n A C h R$ agonists enhance, whereas $\alpha 7 n A C h R$ antagonists reduce, the release of glutamate into the extracellular compartment (Barik and Wonnacott, 2006). Therefore, although a primary effect of KYNA on NMDARs cannot be ruled out based on our present results, we propose that the reductions and elevations of extracellular glutamate described here are caused by fluctuations of endogenously produced KYNA acting through $\alpha 7 n A C h R s$.

Activation of $\alpha 7 \mathrm{nAChRs}$, like activation of NMDARs, increases the performance of experimental animals in the MWM (Duffy et al, 2008; Meyer et al, 1997; Riekkinen and Riekkinen, 1997; Timmermann et al, 2007; Wallace et al, 2011), a task that is extensively used to test hippocampusdependent spatial navigation and reference memory in rodents. On the other hand, reduced activity of either of these two receptors causes cognitive deficits in this paradigm (Boess et al, 2007; Curzon et al, 2006; Morris, 
2006; Wallace et al, 2011). More generally, treatment of experimental animals with glutamate receptor antagonists impairs performance in a variety of cognitive tasks (Ahlander et al, 1999; Hauber and Schmidt, 1989; Karasawa et al, 2008; Venable and Kelly, 1990), whereas endogenous or exogenous glutamate receptor agonists promote cognitive performance (Clem et al, 2008; Lynch et al, 2008; Singer et al, 2010). Although definitive confirmation will require further experimentation and additional brain areas may well be involved (Jo et al, 2007), the present findings suggest that the cognitive impairment caused by elevated KYNA was likely related to reductions in extracellular glutamate in the hippocampus, and that the cognition-enhancing effects of ESBA were the result of elevated extracellular glutamate.

We demonstrated recently that genetic elimination of KAT-II, that is, a chronic reduction in KYNA production, too, causes an elevation in extracellular glutamate levels in the hippocampus. These KAT-II-knockout mice also showed enhanced cognitive abilities and showed increased hippocampal long-term potentiation, providing the first proof-of-concept that a reduction in endogenous KYNA boosts cognition and synaptic plasticity (Potter et al, 2010). In turn, our present results are the first to reveal a similar effect after an acute decrease in endogenous KYNA levels. Notably, this concept has recently stimulated the development of second-generation KAT-II inhibitors, which have shown promising pro-cognitive effects in preliminary studies (Abbott et al, 2010; Chapin et al, 2010).

In summary, the present findings indicate a close association between endogenous KYNA, glutamate, and cognitive performance, and suggest that pharmacological agents that inhibit KAT-II activity in the brain may rapidly enhance cognitive abilities. This targeted approach may constitute an effective, novel strategy to improve cognition not only under normal physiological conditions but also in diseases that are associated with cognitive dysfunctions.

\section{ACKNOWLEDGEMENTS}

This work was supported by USPHS grant NS25296. We thank Dr Robert McMahon for expert assistance with the statistical analyses.

\section{DISCLOSURE}

During the past 3 years, RS has received research support from Mitsubishi-Tanabe (Yokohama, Japan) and BristolMyers-Squibb (Wallingford, CT, USA), and served as a consultant to Merck (West Point, PA, USA). The other authors have nothing to declare.

\section{REFERENCES}

Abbott A, Roberts BM, Turner L, Campbell DW, Schaffer CL, Campbell BM et al (2010). Inhibition of kynurenine aminotransferase II (KAT II) protects against ketamine-induced cognitive impairment and improves spatial working memory. Soc Neurosci Abstr 35: 472.18.

Ahlander M, Misane I, Schott PA, Ogren SO (1999). A behavioral analysis of the spatial learning deficit induced by the NMDA receptor antagonist MK-801 (dizocilpine) in the rat. Neuropsychopharmacology 21: 414-426.
Albuquerque EX, Pereira EF, Alkondon M, Rogers SW (2009). Mammalian nicotinic acetylcholine receptors: from structure to function. Physiol Rev 89: 73-120.

Amori L, Wu HQ, Marinozzi M, Pellicciari R, Guidetti P, Schwarcz R (2009). Specific inhibition of kynurenate synthesis enhances extracellular dopamine levels in the rodent striatum. Neuroscience 159: 196-203.

Bannerman DM, Rawlins JN, Good MA (2006). The drugs don't work-or do they? Pharmacological and transgenic studies of the contribution of NMDA and GluR-A-containing AMPA receptors to hippocampal-dependent memory. Psychopharmacology (Berl) 188: $552-566$.

Baran H, Jellinger K, Deecke L (1999). Kynurenine metabolism in Alzheimer's disease. J Neural Transm 106: 165-181.

Barik J, Wonnacott S (2006). Indirect modulation by alpha7 nicotinic acetylcholine receptors of noradrenaline release in rat hippocampal slices: interaction with glutamate and GABA systems and effect of nicotine withdrawal. Mol Pharmacol 69: 618-628.

Barnes J, Ourselin S, Fox NC (2009). Clinical application of measurement of hippocampal atrophy in degenerative dementias. Hippocampus 19: 510-516.

Boess FG, De Vry J, Erb C, Flessner T, Hendrix M, Luithle J et al (2007). The novel alpha7 nicotinic acetylcholine receptor agonist $N$-[(3R)-1-azabicyclo[2.2.2]oct-3-yl]-7-[2-(methoxy) phenyl]-1-benzofuran-2-carboxamide improves working and recognition memory in rodents. J Pharmacol Exp Ther 321: 716-725.

Carpenedo R, Chiarugi A, Russi P, Lombardi G, Carla V, Pellicciari R et al (1994). Inhibitors of kynurenine hydroxylase and kynureninase increase cerebral formation of kynurenate and have sedative and anticonvulsant activities. Neuroscience 61: 237-243.

Carpenedo R, Pittaluga A, Cozzi A, Attucci S, Galli A, Raiteri M et al (2001). Presynaptic kynurenate-sensitive receptors inhibit glutamate release. Eur J Neurosci 13: 2141-2147.

Chapin DS, Campbell B, Strick C, Kozak R (2010). The impact of a KATII inhibitor on performance in the rat sustained attentional task (SAT) and conditioned avoidance responding (CAR). Soc Neurosci Abstr 35: 472.16.

Chen Y, Meininger V, Guillemin GJ (2009). Recent advances in the treatment of amyotrophic lateral sclerosis. Emphasis on kynurenine pathway inhibitors. Cent Nerv Syst Agents Med Chem 9: 32-39.

Chess AC, Bucci DJ (2006). Increased concentration of cerebral kynurenic acid alters stimulus processing and conditioned responding. Behav Brain Res 170: 326-332.

Chess AC, Landers AM, Bucci DJ (2009). L-Kynurenine treatment alters contextual fear conditioning and context discrimination but not cue-specific fear conditioning. Behav Brain Res 201: 325-331.

Chess AC, Simoni MK, Alling TE, Bucci DJ (2007). Elevations of endogenous kynurenic acid produce spatial working memory deficits. Schizophr Bull 33: 797-804.

Clem RL, Celikel T, Barth AL (2008). Ongoing in vivo experience triggers synaptic metaplasticity in the neocortex. Science 319: 101-104.

Cosi C, Mannaioni G, Cozzi A, Carla V, Sili M, Cavone L et al (2010). G-protein coupled receptor 35 (GPR35) activation and inflammatory pain: studies on the antinociceptive effects of kynurenic acid and zaprinast. Neuropharmacology 60: $1227-1231$.

Curatolo L, Caccia C, Speciale C, Raimondi L, Cini M, Marconi M et al (1996). Modulation of extracellular kynurenic acid content by excitatory amino acids in primary cultures of rat astrocytes. Adv Exp Med Biol 398: 273-276.

Curzon P, Anderson DJ, Nikkel AL, Fox GB, Gopalakrishnan M, Decker MW et al (2006). Antisense knockdown of the rat alpha7 
nicotinic acetylcholine receptor produces spatial memory impairment. Neurosci Lett 410: 15-19.

Duffy S, Labrie V, Roder JC (2008). D-serine augments NMDANR2B receptor-dependent hippocampal long-term depression and spatial reversal learning. Neuropsychopharmacology 33: 1004-1018.

Erhardt S, Blennow K, Nordin C, Skogh E, Lindstrom LH, Engberg $\mathrm{G}$ (2001). Kynurenic acid levels are elevated in the cerebrospinal fluid of patients with schizophrenia. Neurosci Lett 313: 96-98.

Erhardt S, Schwieler L, Emanuelsson C, Geyer M (2004). Endogenous kynurenic acid disrupts prepulse inhibition. Biol Psychiatry 56: 255-260.

Fabian-Fine R, Skehel P, Errington ML, Davies HA, Sher E, Stewart MG et al (2001). Ultrastructural distribution of the alpha7 nicotinic acetylcholine receptor subunit in rat hippocampus. J Neurosci 21: 7993-8003.

Gramsbergen JB, Hodgkins PS, Rassoulpour A, Turski WA, Guidetti P, Schwarcz R (1997). Brain-specific modulation of kynurenic acid synthesis in the rat. J Neurochem 69: 290-298.

Gray R, Rajan AS, Radcliffe KA, Yakehiro M, Dani JA (1996). Hippocampal synaptic transmission enhanced by low concentrations of nicotine. Nature 383: 713-716.

Guidetti P, Amori L, Sapko MT, Okuno E, Schwarcz R (2007a). Mitochondrial aspartate aminotransferase: a third kynurenateproducing enzyme in the mammalian brain. J Neurochem 102: 103-111.

Guidetti P, Hoffman GE, Melendez-Ferro M, Albuquerque EX, Schwarcz R (2007b). Astrocytic localization of kynurenine aminotransferase II in the rat brain visualized by immunocytochemistry. Glia 55: 78-92.

Guillemin GJ, Kerr SJ, Smythe GA, Smith DG, Kapoor V, Armati PJ et al (2001). Kynurenine pathway metabolism in human astrocytes: a paradox for neuronal protection. J Neurochem 78: 842-853.

Guillemin GJ, Smith DG, Kerr SJ, Smythe GA, Kapoor V, Armati PJ et al (2000). Characterisation of kynurenine pathway metabolism in human astrocytes and implications in neuropathogenesis. Redox Rep 5: 108-111.

Hamilton NB, Attwell D (2010). Do astrocytes really exocytose neurotransmitters? Nat Rev Neurosci 11: 227-238.

Han Q, Cai T, Tagle DA, Li J (2010). Structure, expression, and function of kynurenine aminotransferases in human and rodent brains. Cell Mol Life Sci 67: 353-368.

Hauber W, Schmidt WJ (1989). Effects of intrastriatal blockade of glutamatergic transmission on the acquisition of T-maze and radial maze tasks. J Neural Transm Gen Sect 78: 29-41.

Hertz L, Zielke HR (2004). Astrocytic control of glutamatergic activity: astrocytes as stars of the show. Trends Neurosci 27: 735-743.

Hilmas C, Pereira EF, Alkondon M, Rassoulpour A, Schwarcz R, Albuquerque EX (2001). The brain metabolite kynurenic acid inhibits alpha7 nicotinic receptor activity and increases nonalpha7 nicotinic receptor expression: physiopathological implications. J Neurosci 21: 7463-7473.

Jo YS, Park EH, Kim IH, Park SK, Kim H, Kim HT et al (2007). The medial prefrontal cortex is involved in spatial memory retrieval under partial-cue conditions. J Neurosci 27: 13567-13578.

Kalia LV, Kalia SK, Salter MW (2008). NMDA receptors in clinical neurology: excitatory times ahead. Lancet Neurol 7: 742-755.

Kantrowitz JT, Javitt DC (2010). Thinking glutamatergically: changing concepts of schizophrenia based upon changing neurochemical models. Clin Schizophr Relat Psychoses 4: 189-200.

Karasawa J, Hashimoto K, Chaki S (2008). D-serine and a glycine transporter inhibitor improve MK-801-induced cognitive deficits in a novel object recognition test in rats. Behav Brain Res 186: 78-83.

Kessler M, Terramani T, Lynch G, Baudry M (1989). A glycine site associated with $N$-methyl-D-aspartic acid receptors: characterization and identification of a new class of antagonists. J Neurochem 52: 1319-1328.

Konradsson-Geuken A, Gash CR, Alexander K, Pomerleau F, Huettl P, Gerhardt GA et al (2009). Second-by-second analysis of alpha 7 nicotine receptor regulation of glutamate release in the prefrontal cortex of awake rats. Synapse 63: 1069-1082.

Lagostena L, Trocme-Thibierge C, Morain P, Cherubini E (2008). The partial alpha7 nicotine acetylcholine receptor agonist S 24795 enhances long-term potentiation at CA3-CA1 synapses in the adult mouse hippocampus. Neuropharmacology 54: 676-685.

Levin ED, McClernon FJ, Rezvani AH (2006). Nicotinic effects on cognitive function: behavioral characterization, pharmacological specification, and anatomic localization. Psychopharmacology (Berl) 184: 523-539.

Lynch G, Rex CS, Chen LY, Gall CM (2008). The substrates of memory: defects, treatments, and enhancement. Eur J Pharmacol 585: 2-13.

Martin LF, Freedman R (2007). Schizophrenia and the alpha7 nicotinic acetylcholine receptor. Int Rev Neurobiol 78: 225-246.

Meyer EM, Tay ET, Papke RL, Meyers C, Huang GL, de Fiebre CM (1997). 3-[2,4-Dimethoxybenzylidene] anabaseine (DMXB) selectively activates rat alpha7 receptors and improves memoryrelated behaviors in a mecamylamine-sensitive manner. Brain Res 768: 49-56.

Monaghan DT, Bridges RJ, Cotman CW (1989). The excitatory amino acid receptors: their classes, pharmacology, and distinct properties in the function of the central nervous system. Annu Rev Pharmacol Toxicol 29: 365-402.

Moroni F, Cozzi A, Carpendo R, Cipriani G, Veneroni O, Izzo E (2005). Kynurenine 3-mono-oxygenase inhibitors reduce glutamate concentration in the extracellular spaces of the basal ganglia but not in those of the cortex or hippocampus. Neuropharmacology 48: 788-795.

Moroni F, Russi P, Lombardi G, Beni M, Carla V (1988). Presence of kynurenic acid in the mammalian brain. J Neurochem 51: $177-180$.

Morris R (1984). Developments of a water-maze procedure for studying spatial learning in the rat. J Neurosci Methods 11: 47-60.

Morris RG (2006). Theories of hippocampal function. In: Andersen P, Morris R, Amaral D, Bliss T, O'Keefe J (eds). The Hippocampus Book. Oxford University Press: New York. pp 581-713.

Mudo G, Belluardo N, Fuxe K (2007). Nicotinic receptor agonists as neuroprotective/neurotrophic drugs. Progress in molecular mechanisms. J Neural Transm 114: 135-147.

Nemeth H, Toldi J, Vécsei L (2005). Role of kynurenines in the central and peripheral nervous systems. Curr Neurovasc Res 2: 249-260.

O'Connor JC, Andre C, Wang Y, Lawson MA, Szegedi SS, Lestage $\mathrm{J}$ et al (2009). Interferon-gamma and tumor necrosis factor-alpha mediate the upregulation of indoleamine 2, 3-dioxygenase and the induction of depressive-like behavior in mice in response to bacillus Calmette-Guerin. J Neurosci 29: 4200-4209.

Parpura V, Zorec R (2010). Gliotransmission: exocytotic release from astrocytes. Brain Res Rev 63: 83-92.

Parsons CG, Danysz W, Quack G, Hartmann S, Lorenz B, Wollenburg C et al (1997). Novel systemically active antagonists of the glycine site of the $N$-methyl-D-aspartate receptor: electrophysiological, biochemical and behavioral characterization. J Pharmacol Exp Ther 283: 1264-1275.

Pellicciari R, Rizzo RC, Costantino G, Marinozzi M, Amori L, Guidetti P et al (2006). Modulators of the kynurenine pathway of tryptophan metabolism: synthesis and preliminary biological evaluation of (S)-4-(ethylsulfonyl)benzoylalanine, a potent and selective kynurenine aminotransferase II (KAT II) inhibitor. ChemMedChem 1: 528-531. 
Pellicciari R, Venturoni F, Bellocchi D, Carotti A, Marinozzi M, Macchiarulo A et al (2008). Sequence variants in kynurenine aminotransferase II (KAT II) orthologs determine different potencies of the inhibitor S-ESBA. ChemMedChem 3: 1199-1202.

Pereira EF, Hilmas C, Santos MD, Alkondon M, Maelicke A, Albuquerque EX (2002). Unconventional ligands and modulators of nicotinic receptors. J Neurobiol 53: 479-500.

Perkins MN, Stone TW (1982). An iontophoretic investigation of the actions of convulsant kynurenines and their interaction with the endogenous excitant quinolinic acid. Brain Res 247: 184-187.

Potter MC, Elmer GI, Bergeron R, Albuquerque EX, Guidetti P, Wu HQ et al (2010). Reduction of endogenous kynurenic acid formation enhances extracellular glutamate, hippocampal plasticity, and cognitive behavior. Neuropsychopharmacology 35: 1734-1742.

Quarta D, Borycz J, Solinas M, Patkar K, Hockemeyer J, Ciruela F et al (2004). Adenosine receptor-mediated modulation of dopamine release in the nucleus accumbens depends on glutamate neurotransmission and $\mathrm{N}$-methyl-D-aspartate receptor stimulation. J Neurochem 91: 873-880.

Raison CL, Dantzer R, Kelley KW, Lawson MA, Woolwine BJ, Vogt $\mathrm{G}$ et al (2010). CSF concentrations of brain tryptophan and kynurenines during immune stimulation with IFN-alpha: relationship to CNS immune responses and depression. Mol Psychiatry 15: 393-403.

Rassoulpour A, Wu HQ, Ferré S, Schwarcz R (2005). Nanomolar concentrations of kynurenic acid reduce extracellular dopamine levels in the striatum. J Neurochem 93: 762-765.

Richter-Levin G, Canevari L, Bliss TV (1995). Long-term potentiation and glutamate release in the dentate gyrus: links to spatial learning. Behav Brain Res 66: 37-40.

Riekkinen M, Riekkinen Jr P. (1997). Nicotine and D-cycloserine enhance acquisition of water maze spatial navigation in aged rats. Neuroreport 8: 699-703.

Robbins TW, Murphy ER (2006). Behavioural pharmacology: $40+$ years of progress, with a focus on glutamate receptors and cognition. Trends Pharmacol Sci 27: 141-148.

Russi P, Alesiani M, Lombardi G, Davolio P, Pellicciari R, Moroni F (1992). Nicotinylalanine increases the formation of kynurenic acid in the brain and antagonizes convulsions. J Neurochem 59: 2076-2080.

Scheff SW, Price DA (2006). Alzheimer's disease-related alterations in synaptic density: neocortex and hippocampus. J Alzheimers Dis 9: 101-115.

Schwarcz R, Du F, Schmidt W, Turski WA, Gramsbergen JB, Okuno E et al (1992). Kynurenic acid: a potential pathogen in brain disorders. Ann NY Acad Sci 648: 140-153.

Schwarcz R, Pellicciari R (2002). Manipulation of brain kynurenines: glial targets, neuronal effects, and clinical opportunities. J Pharmacol Exp Ther 303: 1-10.

Schwarcz R, Rassoulpour A, Wu HQ, Medoff D, Tamminga CA, Roberts RC (2001). Increased cortical kynurenate content in schizophrenia. Biol Psychiatry 50: 521-530.

Shepard PD, Joy B, Clerkin L, Schwarcz R (2003). Micromolar brain levels of kynurenic acid are associated with a disruption of auditory sensory gating in the rat. Neuropsychopharmacology 28: $1454-1462$.

Shibata K (1988). Fluorimetric micro-determination of kynurenic acid, an endogenous blocker of neurotoxicity, by high-performance liquid chromatography. J Chromatogr 430: 376-380.

Singer HS, Morris C, Grados M (2010). Glutamatergic modulatory therapy for Tourette syndrome. Med Hypotheses 74: 862-867.

Speciale C, Wu HQ, Gramsbergen JB, Turski WA, Ungerstedt U, Schwarcz R (1990). Determination of extracellular kynurenic acid in the striatum of unanesthetized rats: effect of aminooxyacetic acid. Neurosci Lett 116: 198-203.

Swartz KJ, During MJ, Freese A, Beal MF (1990). Cerebral synthesis and release of kynurenic acid: an endogenous antagonist of excitatory amino acid receptors. J Neurosci 10: 2965-2973.

Tamminga CA, Stan AD, Wagner AD (2010). The hippocampal formation in schizophrenia. Am J Psychiatry 167: 1178-1193.

Thomsen MS, Hansen HH, Timmerman DB, Mikkelsen JD (2010). Cognitive improvement by activation of alpha7 nicotinic acetylcholine receptors: from animal models to human pathophysiology. Curr Pharm Des 16: 323-343.

Timmermann DB, Gronlien JH, Kohlhaas KL, Nielsen EO, Dam E, Jorgensen TD et al (2007). An allosteric modulator of the alpha7 nicotinic acetylcholine receptor possessing cognition-enhancing properties in vivo. J Pharmacol Exp Ther 323: 294-307.

Turski WA, Nakamura M, Todd WP, Carpenter BK, Whetsell Jr WO., Schwarcz R (1988). Identification and quantification of kynurenic acid in human brain tissue. Brain Res 454: 164-169.

Varasi M, Della Torre A, Heidempergher F, Pevarello P, Speciale C, Guidetti $P$ et al (1996). Derivatives of kynurenine as inhibitors of rat brain kynurenine aminotransferase. Eur J Med Chem 31: 11-21.

Venable N, Kelly PH (1990). Effects of NMDA receptor antagonists on passive avoidance learning and retrieval in rats and mice. Psychopharmacology (Berl) 100: 215-221.

Wallace TL, Callahan PM, Tehim A, Bertrand D, Tombaugh G, Wang S et al (2011). RG3487, a novel nicotinic alpha7 receptor partial agonist, improves cognition and sensorimotor gating in rodents. J Pharmacol Exp Ther 336: 242-253.

Wang J, Simonavicius N, Wu X, Swaminath G, Reagan J, Tian H et al (2006). Kynurenic acid as a ligand for orphan $G$ proteincoupled receptor GPR35. J Biol Chem 281: 22021-22028.

Wu HQ, Pereira EF, Bruno JP, Pellicciari R, Albuquerque EX, Schwarcz R (2010). The astrocyte-derived alpha7 nicotinic receptor antagonist kynurenic acid controls extracellular glutamate levels in the prefrontal cortex. J Mol Neurosci 40: 204-210.

Wu HQ, Rassoulpour A, Schwarcz R (2007). Kynurenic acid leads, dopamine follows: a new case of volume transmission in the brain? J Neural Transm 114: 33-41.

Zmarowski A, Wu HQ, Brooks JM, Potter MC, Pellicciari R, Schwarcz $\mathrm{R}$ et al (2009). Astrocyte-derived kynurenic acid modulates basal and evoked cortical acetylcholine release. Eur J Neurosci 29: 529-538.

Supplementary Information accompanies the paper on the Neuropsychopharmacology website (http://www.nature.com/npp) 